\title{
Charging Effects on SEM/SIM Contrast of Metal/Insulator System in Various Metallic Coating Conditions
}

\author{
Ki Hyun Kim ${ }^{1, *}$, Zentaro Akase ${ }^{1}$, Toshiaki Suzuki ${ }^{2}$ and Daisuke Shindo ${ }^{1}$ \\ ${ }^{1}$ Institute of Multidisciplinary Research for Advanced Materials, Tohoku University, Sendai 980-8577, Japan \\ ${ }^{2}$ JEOL Ltd., Tokyo 196-8558, Japan
}

\begin{abstract}
Scanning electron microscope (SEM) and scanning ion microscope (SIM) observations were performed to investigate the charging effect and the related contrast variation for images of conductive and non-conductive specimens under electron and $\mathrm{Ga}^{+}$ion beam irradiations. The contrast variation in the specimens was investigated by changing the coating conditions. It was found that the contrast in conductive specimens was basically caused by differences in atomic number and that the charging effect in conductive specimens is generally smaller than that in nonconductive specimens. On the other hand, the SEM contrast of non-conductive specimens varied widely, depending on the coating conditions. These contrast variations were found to be caused by negative charges accumulated on the surface of the specimens. The SIM contrast of nonconductive specimens changed to dark when the coating condition was insufficient. The contrast variations were found to be caused by the accumulation of positive charges on the surface of specimens. [doi:10.2320/matertrans.M2010034]
\end{abstract}

(Received January 27, 2010; Accepted March 26, 2010; Published May 19, 2010)

Keywords: charging, osmium (Os) coating, scanning electron microscopy (SEM)/scanning ion microscopy (SIM) contrast

\section{Introduction}

Electron/ion microscopy techniques have been widely employed for the characterization of various specimens such as metals, insulators, and polymers. Among the many microscopic techniques, scanning electron microscopy (SEM) and focused ion beam (FIB) techniques have been extensively employed in the fields of materials science. ${ }^{1,2)}$ The formation of image contrast by these techniques is attributed to secondary electrons excited on the specimen surface by the incident electron and $\mathrm{Ga}^{+}$ion beam irradiations. These electron- and ion-induced secondary electron images can be used for surface analysis of various specimens. Therefore, the yield of secondary electrons emitted from specimens is an important factor for reliable interpretations of image contrast. ${ }^{3,4)}$

In recent years, many studies have focused on interpreting SEM and scanning ion microscopy (SIM) image contrasts. ${ }^{5-7)}$ In the case of conductive specimen, the dependence of secondary electron yield on the atomic number of a specimen has been investigated. The results of these studies have shown that the secondary electron yield in SEM tends to increase with an increase in the atomic number. On the other hand, the secondary electron yield in SIM decreases with an increase in the atomic number. It has been explained that this tendency is caused by the difference between the penetration depths of ion and electron beams. ${ }^{5-7)}$

When the SEM or SIM contrast of a non-conductive specimen is interpreted, the charging effect arising from electron/ion irradiation is unavoidable. In general, the charging effect is caused by the accumulation of static electric charges on the specimen surface, which results in many problems. $^{8-11)}$ For example, the negative charge would flip the secondary electron from the surface. The negative charge would also reduce the landing energy of the incident electrons, and it would increase the field between

*Present address: Nanostructures Research Laboratory, Japan Fine Ceramics Center, Nagoya 456-8587, Japan the surface and secondary electron detector. On the other hand, positive charge would re-collect the secondary electron. ${ }^{12)}$ In order to discuss the secondary electron emission from non-conductive specimen quantitatively, a model have been presented. ${ }^{13,14)}$ It shows the yield is not only a function of the chemical composition but also functions of the specimen structure, temperature and the irradiation conditions. Consequently, reliable evaluation of SEM/SIM images of non-conductive specimens is difficult. For reliable analysis, in addition to the suppression of the charging effect through the use of a metallic coating, evaluating how the charging effect is suppressed by the metallic coating is also important.

In this study, we investigated the change of SEM and SIM image contrasts of conductive and non-conductive specimens with various metallic coating conditions in order to evaluate how the charging effect appears on those specimens. We selected an $\mathrm{Al}_{2} \mathrm{O}_{3}$ sintered body, tungsten (W), and aluminum $(\mathrm{Al})$ as typical non-conductive and conductive specimens. Those specimens were prepared with osmium (Os) coating under several conditions.

\section{Experimental Methods}

Figure 1(a) shows a schematic illustration indicating the coating conditions of the specimens. Here, an $\mathrm{Al}_{2} \mathrm{O}_{3}$ sintered body was prepared by a pressureless sintering method at $1600^{\circ} \mathrm{C}$ using commercial $\mathrm{Al}_{2} \mathrm{O}_{3}$ powder (AKP50, Sumimoto). ${ }^{15)}$ For conductive specimens, commercial aluminum and tungsten were used. These specimens were mounted in epoxy resin which was fixed at room temperature for $12 \mathrm{~h}$; the mounted specimens were then sectioned using a diamond saw and thinned using $\mathrm{SiC}$ grinding paper. The size of the prepared specimens is indicated in Fig. 1(a). The specimens were coated with $2 \mathrm{~nm}$ of Os using a commercial Os plasma coater (OPC60A, Filgen Inc.). Figures 1(a-I)(a-III) show the coating conditions for specimens corresponding to full-coating, side-coating, and no-coating, respectively. 
(a)

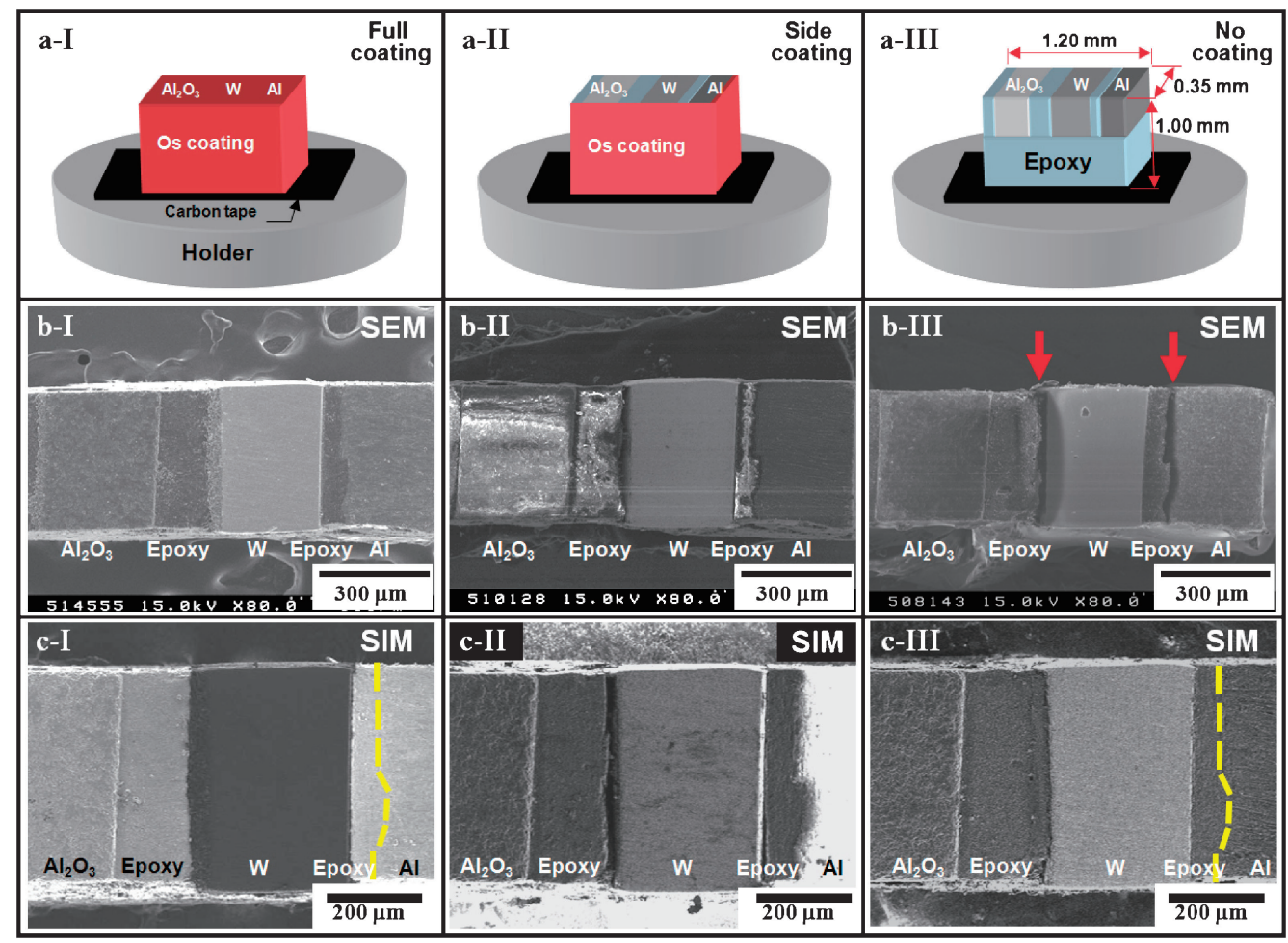

Fig. 1 (a) Schematic illustrations corresponding to the Os coating conditions of specimens. The Os coating layers are indicated by red. (b) SEM images showing the contrast variation for conductive and non-conductive specimens depending on the coating conditions. The accelerating voltage was $15 \mathrm{kV}$. (c) SIM images showing the contrast variation for conductive and non-conductive specimens depending on the coating conditions. The accelerating voltage and intensity of the Ga ion beam were $30 \mathrm{kV}$ and $50 \mathrm{pA}$, respectively. The yellow dotted line indicates the boundary between the epoxy and aluminum.

SEM and SIM observations were carried out using S4100L, JEM-9310FIB and JIB-4500 with the detectors located at the lateral positions. The SEM and SIM images were obtained at accelerating voltages of 15 and $30 \mathrm{kV}$, respectively. For the SEM and SIM observations, the contrast variation in the specimens was investigated as a function of the coating conditions.

\section{Results and Discussion}

Figures 1(b) and (c) show SEM and SIM images depending on the metallic coating conditions schematically illustrated in Fig. 1(a). Figures 1(b-I) and (c-I) represent SEM and SIM images of the full-coating condition, respectively. Under the full-coating condition, tungsten provided the brightest SEM image and the darkest SIM image. This result can be explained as follows: in the SEM contrast, the yields of secondary electrons in the heavy elements are generally larger than that of light elements, since heavy elements contain large number of electrons. Therefore the SEM contrast of heavy elements such as tungsten tends to be brighter than that of light elements, such as aluminum. On the other hand, in the case of SIM, due to the large interaction between the ion beam and atoms in the material surface, penetration depth is much smaller in the heavy elements than the light elements. Thus the yields of secondary electrons from the heavy elements are small, resulting in the dark SIM contrast, as reported previously. ${ }^{5-7)}$ These results show that the accumulation of static electric charges on the specimens is completely prevented by fully coating the specimen with
Os, as shown in Figs. 2(a) and (d). Therefore, in the fullcoating condition, the SEM and SIM image contrasts of all specimens were not affected by the charging effect.

The SEM images for side-coating and no-coating conditions are shown in Figs. 1(b-II) and (b-III), respectively. The contrast variation between conductive specimens such as tungsten and aluminum is similar to the case of the fullcoating as shown in Fig. 1(b-I). On the other hand, the contrast of the non-conductive specimens shows two significant differences in comparison with the case of conductive specimens. The first difference is the dependence of contrast at central portion of specimen on the coating conditions. In the side-coating condition, the contrast at central portions of non-conductive specimens became bright, as shown in Fig. 1(b-II). And in the no-coating condition, those portions became dark again, as shown in Fig. 1(b-III). This dependence can be interpreted by the following explanation. In the case of side-coating condition, the edges of the nonconductive specimen are observed to be dark, because negative charges do not accumulate on the edges of the non-conductive specimens due to the side coating of Os. On the other hand, the central portions of the non-conductive specimens have bright contrast because those portions are negatively charged. Since the non-conductive specimens are charged negatively, secondary electrons are more easily emitted due to the repulsive force, as shown in Fig. 2(b). In the case of no-coating condition, it is considered that the accumulation of negative charge on the surface was severer than that in the case of side-coating condition. In this case, it is considered that the incident electron beam tends to be 


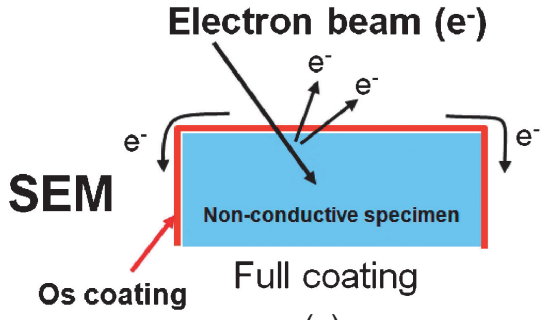

(a)

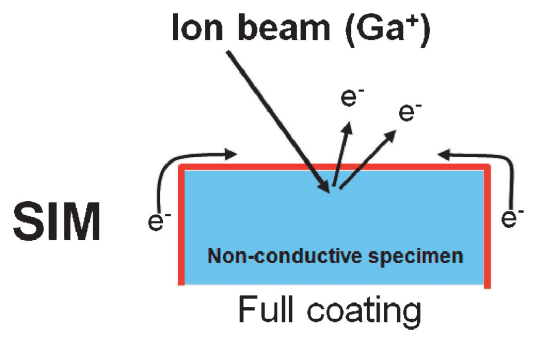

(d)

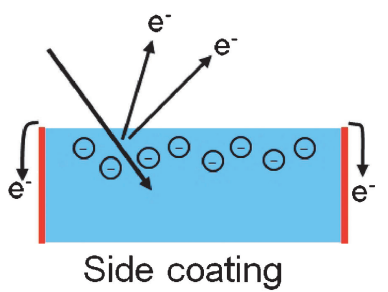

(b)

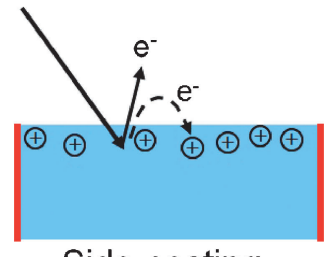

Side coating

(e)

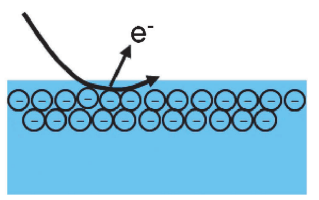

No coating

(c)

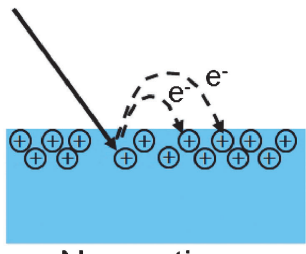

No coating

(f)

Fig. 2 Schematic illustration of secondary electron emission for non-conductive specimens depending on the coating conditions. (a)-(c) Schematic illustrations for an SEM under electron beam irradiation. (d)-(f) Schematic illustrations for an SIM under Ga ion beam irradiation. The Os coating layers are indicated by red lines.

deflected due to the severe charge accumulation on the surface as shown in Fig. 2(c). Thus, the number of the secondary electrons emitted from the specimen may decrease due to the decrease of incident electrons landing to the specimen. The second difference is that abnormal dark contrasts appeared in tungsten and aluminum, as indicated by red arrows in Fig. 1(b-III). It is noted that the edge part of the abnormal dark contrast is narrow, and the middle part is wide. It is considered that the edges of abnormal dark contrasts were partially compensated for by leakage of electrons into the substrate (earth). On the other hand, in the middle region of the abnormal dark contrasts, the secondary electrons were affected by a severe charging effect in epoxy. Since the electron beam is scanned on the specimen from the left to the right, it is considered that the regions on the right of epoxy tend to be more affected by the charging effect. The width of the dark contrast is small. This may be due to the thin thickness of the epoxy near the aluminum. In order to understand the change of SEM contrast due to the charging effect and abnormal contrast accurately, further experiment with electric field analysis will be carried out in future.

The SIM images for side-coating and no-coating conditions are shown in Figs. 1(c-II) and (c-III), respectively. In the side-coating condition shown in Fig. 1(c-II), the contrast variation between conductive specimens is similar to the case of the full-coating as shown in Fig. 1(c-I). However, in the no-coating condition shown in Fig. 1(c-III), the contrasts of tungsten became brighter than that of aluminum. This contrast variation is different from those cases in the fullcoating and the side-coating conditions. The reason can be explained as follows. In the no-coating condition, the conductive specimens are isolated from the substrate electrically, as shown in Fig. 1(a-III). Thus, positive charges accumulate even on the surface of conductive specimens due to the $\mathrm{Ga}^{+}$ion implantation. If there is no accumulation of positive charges, the penetration depth in aluminum is wider than that in tungsten. But the accumulation of positive charges limits the penetration depths of both specimens to the surface region only. Considering that tungsten has more electrons than aluminum as discussed above, the contrast of tungsten becomes relatively brighter than that of aluminum due to the higher probability of secondary electron emission. On the other hand, in the case of non-conductive specimens, the contrasts in the side-coating and no-coating conditions became darker than that in the full-coating condition. It is considered that these variations are attributed to positive charging, since for SIM images of the side-coating and nocoating conditions, non-conductive specimens are positively charged due to $\mathrm{Ga}^{+}$ion implantation. Consequently, the secondary electrons emitted from the specimen are attracted to the surface of the positively charged specimen, as shown in Figs. 2(e) and (f) which is consistent with the discussion in the reference. ${ }^{3)}$

Finally, we should note that the contrast variations noted above were found to have the same tendency when we observed specimens with SEM at an accelerating voltage of $10 \mathrm{kV}$ and with SIM at accelerating voltages of 15 and $20 \mathrm{kV}$.

\section{Conclusion}

The findings of this study on the charging effect and the related contrast variation under electron and ion beam irradiation can be summarized as follows:

(1) The contrast of SEM and SIM images is basically caused by the difference in atomic number of the specimens because the atomic number is related to the penetration depth of conductive specimens for electron and ion irradiation. However, when the coating was insufficient, the contrast of non-conductive specimens could not be explained by the atomic number. The contrast was found to strongly depend on the charging effect and metallic coating conditions.

(2) Depending on the state of coating conditions, the contrasts of non-conductive specimens in the SEM images were changed. This result indicates that these contrast 
variations are caused by accumulated negative charges on the surface of specimens. On the other hand, the contrasts in the SIM image became dark when the charging effect occurred. This result reveals that the contrast of non-conductive specimens can be attributed to positive charges accumulated on the surface of specimens due to $\mathrm{Ga}^{+}$ion beam irradiation.

\section{Acknowledgements}

The authors are grateful to Mr. A. Ono and Mr. S. Ikeda for their useful discussion.

\section{REFERENCES}

1) S. Reyntjens and R. Puers: J. Micromech. Microeng. 11 (2001) 287300.

2) Z. L. Wang, Y. Liu and Z. Zhang: Handbook of nanophase and nanostructured materils Volume II: Characterization, (Kluwer Academic/Plenum Publishers, New York, 2003).
3) L. A. Giannuzzi and F. A. Stevie: Introduction to focused ion beams: instrumentation, theory, techniques and practice, (Springer, New York, 2004) p. 258.

4) H. Seiler: J. Appl. Phys. 54 (1983) 1100R1-R18.

5) Y. Sakai, T. Yamada, T. Suzuki, T. Sato, H. Itoh and T. Ichinokawa: Appl. Phys. Lett. 73 (1998) 611-613.

6) Y. Sakai, T. Yamada, T. Suzuki and T. Ichinokawa: Appl. Surf. Sci. 144-145 (1999) 96-100.

7) T. Suzuki, N. Endo, M. Shibata, S. Kamasaki and T. Ichinokawa: J. Vac. Sci. Technol. A 22 (2004) 49-52.

8) K. Ura and S. Aoyagi: J. Electron Microsc. 49 (2000) 157-162.

9) K. Ura: J. Electron Microsc. 47 (1998) 143-147.

10) M. Fukuda, S. Tomimatsu, H. Shichi and K. Umemura: Microelectron. Eng. 78-79 (2005) 22-28.

11) K. H. Kim, J. J. Kim, T. Suzuki and D. Shindo: J. Electron Microsc. 57 (2008) 53-57.

12) D. C. Joy and C. S. Joy: Micron 27 (1996) 247-263.

13) J. Cazaux: Ultramicrosc. 108 (2008) 1645-1652.

14) J. Cazaux: Microsc. Microanal. 10 (2004) 670-684.

15) B. T. Lee, I. C. Kang, S. H. Cho and H. Y. Song: J. Am. Ceram. Soc. 88 (2005) 2262-2266. 